\section{Endoscopic Diagnosis of a Duodenocolic Fistula Due to a Non-Hodgkin's Lymphoma in a Patient with AIDS}

The occurrence of an enterocolic fistula, as complication of a highly malignant AIDS-associated lymphoma, is uncommon.

A 45-year-old man with AIDS came to our endoscopy department in May 1995 with weight loss, mild fever, epigastric pain, and diarrhea. Upper gastrointestinal endoscopy showed a large duodenal ulcer (Figure 1 a). Histopathologic examination of the biopsy specimens found a highly malignant B-cell lymphoma. The colonoscopy revealed a large ulcer of the right colic angle, approaching the lesion seen in the duodenum. Surprisingly, a few centimeters further on, the endoscope was located in the stomach. Shortly thereafter, an upper gastrointestinal endoscopy confirmed the fistula between the duodenum and the colon (Figure 1 b), which was also seen on esophagogastroduodenal radiographs (Figure 2). Because the underlying disease was quite advanced (CD4 cell count: $10 / \mathrm{mm}^{3}$ ), cephalic duodenopancreatectomy was not undertaken. Right colectomy and distal gastrectomy were performed. Histopathological examination of the resected specimens confirmed the duodenal location of a highly malignant Bcell lymphoma, and the duodenocolic fistula. One month after surgery, the patient was treated with combined chemotherapy.

Untreated gastrointestinal lymphomas in patients with AIDS have been reported in association with enterocolic fistula in only one case (1). A variety of fistulas have been described in patients with lymphomas, but no information was provided on their HIV status $(2,3)$. In patients with AIDS, the digestive tract manifestations (abdominal pain, diarrhea) are more commonly caused by opportunistic infections. In our patient, an opportunistic infection of the gastrointestinal tract was microbiologically and serologically excluded, leading to gastrointestinal endoscopies and subsequent duodenal lymphoma diagnosis. Our patient's duodenal lymphoma was characterized by massive tumorous involvement of adjacent tissues, extensive necrosis, and an absence of a desmoplastic reaction. It is speculated that desmoplastic reaction, often observed in carcinomas, to a certain extent safeguards against fistulization (4). These features and the anatomic vicinity of the right colic angle, probably promoted the occurrence of a duodenocolic fistula. In patients with AIDS, the presence of abdominal pain, diarrhea, and systemic symptoms, should lead more frequently to gastrointestinal endoscopic examinations in order to detect a gastrointestinal lymphoma as early as possible, even if the prognosis remains poor (5).

H. Bécheur', C. Piketty 2, F. Bloch', D. Levoir', J. M. Aubertin', J. L. Bouillot ${ }^{3}$, J. P. Petite'

' Dept. of Hepatogastroenterology, Broussais Hospital, Paris, France

${ }^{2}$ Dept, of Immunology, Broussais Hospital, Paris, France

${ }^{3}$ Dept. of Digestive Surgery, Broussais Hospital, Paris, France

\section{References}

1. Bruck B, Dette S, Kaiserling E, et al. A fistula between the rectosigmoid junction and the ileum as a complication of highly malignant AIDS-associated lymphoma. Dtsch Med Wochenschr 1991; 116: 1911-4.

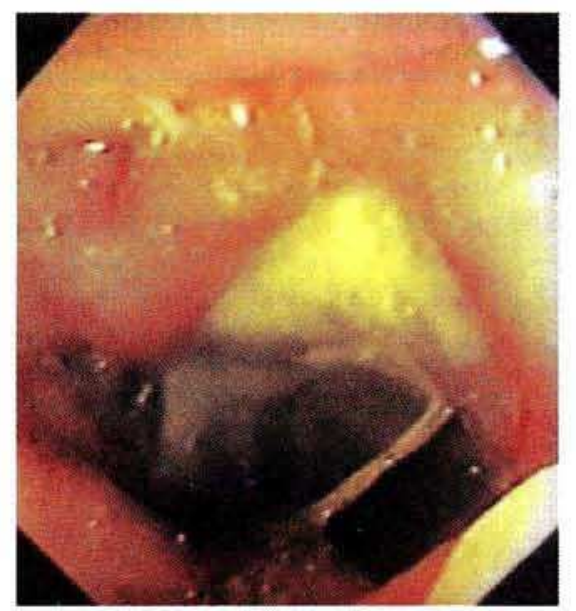

Figure 1a: Large duodenal ulcer. The biopsy specimen revealed a highly malignant B-cell lymphoma.

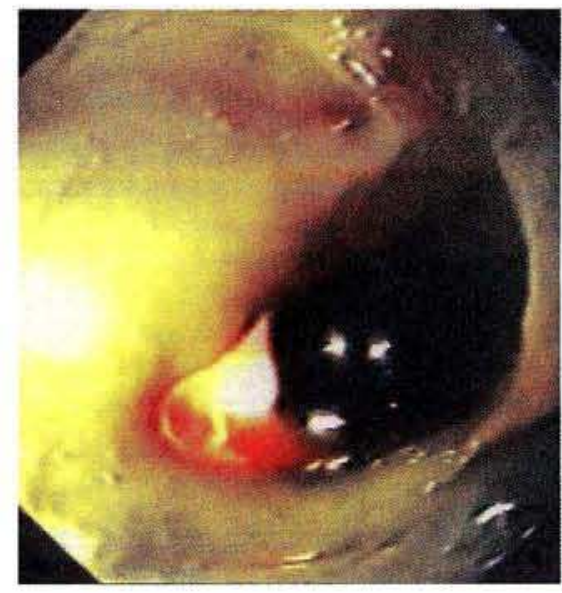

Figure $1 \mathrm{~b}$ : Visualization of the gastroscope through the colonoscope: endoscopic diagnosis of a fistula between the duodenum and the right colic angle.

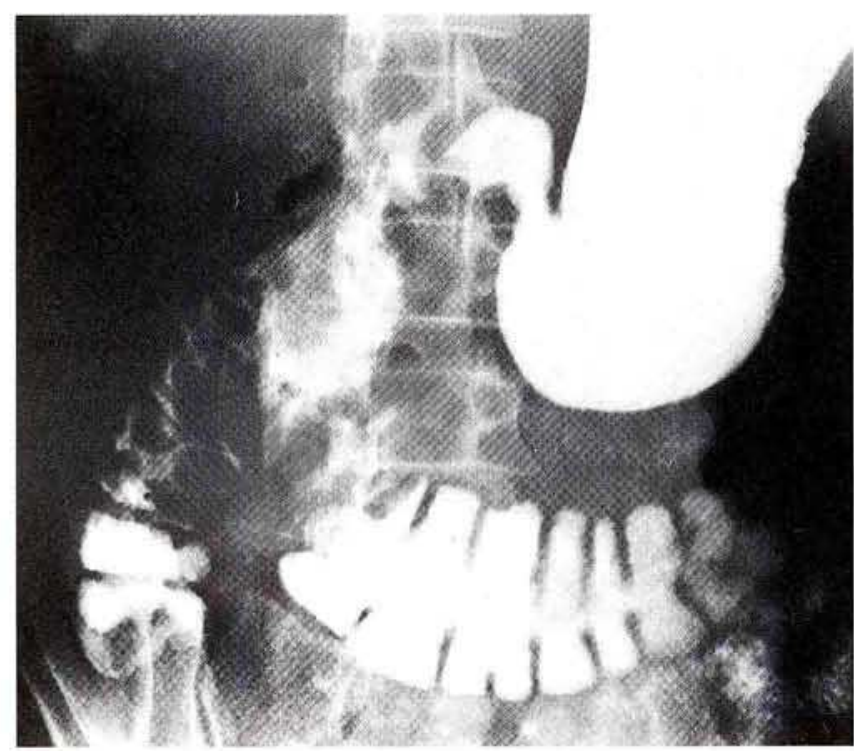

Figure 2: Esophagogastroduodenal radiograph: confirmation of the duodenocolic fistula. 
2. Bubenik O, Lopez MJ, Greco AO, et al. Gastrosplenic fistula following successful chemotherapy for disseminated histiocytic lymphoma. Cancer 1983; 52: 994-6.

3. Harris NL, Aisenberg AC, Meyer JE, et al. Diffuse large cell (histiocytic) lymphoma of the spleen: clinical and pathologic characteristics of ten cases. Cancer 1984; 54: 2460-7.

4. Naschitz JE, Yeshurun D, Horovitz IL, et al. Spontaneous colosplenic fistula complicating immunoblastic lymphoma. Dis Colon Rectum 1986; 29: 521-3.

5. Levine AM, Sullivan-Halley J, Pike MC, et al. HIV-related lymphoma: prognostic factors predictive of survival. Cancer 1991; 68: 2466-72.
Corresponding Author

H. Bécheur, M. D.

Dept. of Hepatogastroenterology

Hôpital Broussais

96, rue Didot

75674 Paris Cédex 14

France

Fax: $+33-1-43959256$ 